\title{
The Effect of Steric Hindrance on the Association of Telluradiazoles through Te-N Secondary Bonding Interactions
}

\author{
Anthony F. Cozzolino, James F. Britten, and Ignacio Vargas-Baca,* \\ Department of Chemistry, McMaster University, \\ 1280 Main Street West, Hamilton, Ontario, Canada L8S 4M1. \\ vargas@chemistry.mcmaster.ca.
}

Supporting Information 
Table S1. Selected bond distances and angles $\left[{ }^{\AA},{ }^{\circ}\right]$ for $\mathbf{4 c}, 5$ and $\mathbf{5} \cdot \mathbf{D M S O}$.

\begin{tabular}{|c|c|c|c|}
\hline & $4 c$ & 5 & 5·DMSO \\
\hline $\mathrm{N}(1)-\mathrm{C}(1)$ & 1.31(1) & $1.30(1)$ & $1.311(7)$ \\
\hline $\mathrm{N}(2)-\mathrm{C}(6)$ & $1.32(1)$ & $1.31(1)$ & $1.320(7)$ \\
\hline $\mathrm{N}(3)-\mathrm{C}(7)$ & $1.33(1)$ & - & - \\
\hline $\mathrm{N}(4)-\mathrm{C}(12)$ & 1.31(1) & - & - \\
\hline $\mathrm{C}(1)-\mathrm{C}(2)$ & $1.45(1)$ & $1.42(1)$ & $1.449(7)$ \\
\hline$C(7)-C(8)$ & $1.40(1)$ & - & - \\
\hline$C(2)-C(3)$ & $1.35(1)$ & $1.37(1)$ & $1.363(8)$ \\
\hline$C(8)-C(9)$ & $1.37(1)$ & - & - \\
\hline$C(3)-C(4)$ & $1.46(1)$ & $1.43(1)$ & $1.434(9)$ \\
\hline$C(9)-C(10)$ & $1.43(1)$ & - & - \\
\hline$C(4)-C(5)$ & $1.32(1)$ & $1.34(1)$ & $1.360(7)$ \\
\hline$C(10)-C(11)$ & $1.37(1)$ & - & - \\
\hline$C(5)-C(6)$ & $1.43(1)$ & $1.43(1)$ & $1.444(7)$ \\
\hline$C(11)-C(12)$ & $1.44(1)$ & - & - \\
\hline$C(1)-C(6)$ & $1.46(1)$ & $1.51(1)$ & $1.474(8)$ \\
\hline$C(7)-C(12)$ & $1.48(1)$ & - & - \\
\hline $\mathrm{C}(2)-\mathrm{Br}(1)$ & - & $1.891(8)$ & $1.891(6)$ \\
\hline$C(5)-B r(2)$ & - & $1.896(9)$ & $1.891(6)$ \\
\hline $\mathrm{O}(1)-\mathrm{S}(1)$ & - & - & $1.514(5)$ \\
\hline$S(1)-C(7)$ & - & - & $1.789(6)$ \\
\hline$S(1)-C(8)$ & - & - & $1.792(7)$ \\
\hline $\mathrm{Te}(1)-\mathrm{N}(1)-\mathrm{C}(1)$ & 109.3(5) & 109.6(6) & 109.3(4) \\
\hline $\mathrm{Te}(2)-\mathrm{N}(3)-\mathrm{C}(7)$ & $111.1(5)$ & - & - \\
\hline $\mathrm{Te}(1)-\mathrm{N}(2)-\mathrm{C}(6)$ & $112.2(5)$ & $110.9(6)$ & $109.7(4)$ \\
\hline $\mathrm{Te}(2)-\mathrm{N}(4)-\mathrm{C}(12)$ & $110.3(5)$ & - & - \\
\hline $\mathrm{N}(1)-\mathrm{C}(1)-\mathrm{C}(6)$ & $119.6(8)$ & $118.3(8)$ & $118.6(5)$ \\
\hline $\mathrm{N}(3)-\mathrm{C}(7)-\mathrm{C}(12)$ & $115.6(8)$ & - & - \\
\hline $\mathrm{N}(2)-\mathrm{C}(6)-\mathrm{C}(1)$ & $115.3(8)$ & $115.7(8)$ & $117.3(5)$ \\
\hline $\mathrm{N}(4)-\mathrm{C}(12)-\mathrm{C}(7)$ & $118.9(8)$ & - & - \\
\hline$C(1)-C(2)-C(3)$ & $119.7(8)$ & $122.5(8)$ & $121.6(5)$ \\
\hline$C(7)-C(8)-C(9)$ & $120.9(8)$ & - & - \\
\hline$C(2)-C(3)-C(4)$ & $120.5(9)$ & $119.8(9)$ & $119.5(5)$ \\
\hline$C(8)-C(9)-C(10)$ & $120.7(8)$ & - & - \\
\hline$C(3)-C(4)-C(5)$ & $122.5(9)$ & 121.1(9) & 122.1(6) \\
\hline$C(9)-C(10)-C(11)$ & $120.9(8)$ & - & - \\
\hline$C(4)-C(5)-C(6)$ & $119.5(9)$ & $122.6(8)$ & $120.8(5)$ \\
\hline $\mathrm{C}(10)-\mathrm{C}(11)-\mathrm{C}(12)$ & $120.3(8)$ & - & - \\
\hline$C(5)-C(6)-C(1)$ & 119.4(8) & $117.0(8)$ & $117.8(5)$ \\
\hline$C(11)-C(12)-C(7)$ & $117.7(8)$ & - & - \\
\hline$C(6)-C(1)-C(2)$ & $118.4(8)$ & $116.9(8)$ & $118.1(5)$ \\
\hline $\mathrm{C}(12)-\mathrm{C}(7)-\mathrm{C}(8)$ & $119.4(8)$ & & \\
\hline$C(1)-C(2)-B r(1)$ & - & $117.3(7)$ & $119.0(4)$ \\
\hline$C(6)-C(5)-B r(2)$ & - & $117.0(7)$ & 119.1(4) \\
\hline
\end{tabular}


Table 2. Supramolecular bond distances and angles $\left[\AA{ }^{\circ}\right]$ for $\mathbf{4 c}$.

\begin{tabular}{ccc}
\hline & Experimental & Calculated \\
\hline $\mathrm{Te}(1)-\mathrm{N}(3)$ & $2.682(7)$ & 2.690 \\
$\mathrm{Te}(2)-\mathrm{N}(2)$ & $2.720(7)$ & \\
$\mathrm{Te}(1)-\mathrm{N}(4)^{\mathrm{a}}$ & $2.714(6)$ & \\
$\mathrm{Te}(2)^{\mathrm{a}}-\mathrm{N}(1)$ & $2.688(6)$ & \\
$\mathrm{N}(2)-\mathrm{Te}(1)-\mathrm{N}(3)$ & $69.7(3)$ & \\
$\mathrm{N}(3)-\mathrm{Te}(2)-\mathrm{N}(2)$ & $68.6(2)$ & \\
$\mathrm{N}(1)-\mathrm{Te}(1)-\mathrm{N}(4)^{\mathrm{a}}$ & $68.8(2)$ & \\
$\mathrm{N}(4)^{\mathrm{a}}-\mathrm{Te}(2)^{\mathrm{a}}-\mathrm{N}(1)$ & $69.5(2)$ & \\
$\mathrm{N}(1)-\mathrm{Te}(1)-\mathrm{N}(3)$ & $153.3(2)$ & \\
$\mathrm{N}(4)-\mathrm{Te}(2)-\mathrm{N}(2)$ & $152.6(2)$ & \\
$\mathrm{N}(2)-\mathrm{Te}(1)-\mathrm{N}(4)^{\mathrm{a}}$ & $152.5(2)$ & \\
$\mathrm{N}(3)^{\mathrm{a}}-\mathrm{Te}(2)^{\mathrm{a}}-\mathrm{N}(1)$ & $153.4(2)$ & \\
${ }^{\mathrm{a}} \mathrm{x}-1 / 2,+\mathrm{y}+1 / 2,+\mathrm{Z}$ & &
\end{tabular}

Table 3. Supramolecular bond distances and angles $\left[\AA{ }^{\circ}{ }^{\circ}\right]$ for $\mathbf{5}$.

\begin{tabular}{cccc}
\hline & Experimental & Calculated for $\mathbf{5}_{\mathbf{2}}$ & Calculated for $\mathbf{5 - 5}_{\mathbf{2}} \mathbf{- 5}$ \\
\hline $\mathrm{Te}(1)-\mathrm{N}(2)^{\mathrm{a}}$ & $2.697(8)$ & 2.620 & 2.663 \\
$\mathrm{~N}(2)-\mathrm{Te}(1)-\mathrm{N}(2)^{\mathrm{a}}$ & $71.1(3)$ & 71.5 & 71.4 \\
$\mathrm{~N}(2)-\mathrm{Te}(1)-\mathrm{N}(2)^{\mathrm{a}}$ & $156.6(3)$ & 156.4 & 156.3 \\
$\mathrm{Br}(1)-\mathrm{Br}(2)^{\mathrm{b}}$ & $3.506(2)$ & & 3.500 \\
$\mathrm{Te}(1)-\mathrm{Br}(1)^{\mathrm{c}}$ & $3.683(1)$ & & 3.636 \\
$\mathrm{~N}(1)-\mathrm{Te}(1)-\mathrm{Br}(1)^{\mathrm{c}}$ & $88.0(2)$ & & 17.9 \\
$\mathrm{~N}(2)-\mathrm{Te}(1)-\mathrm{Br}(1)^{\mathrm{c}}$ & $170.9(2)$ & & 3.671 \\
$\mathrm{Te}(1)-\mathrm{Br}(2)^{\mathrm{a}}$ & $3.605(1)$ & & \\
${ }^{\mathrm{a}}{ }^{\mathrm{c}} \mathrm{x},-\mathrm{y}+1,-\mathrm{z}$ & & & \\
${ }^{\mathrm{b}}-\mathrm{x}-1,+\mathrm{y}-1 / 2,-\mathrm{z}-1 / 2$ & & & \\
${ }^{\mathrm{c}} \mathrm{x}-1,-\mathrm{y}+1 / 2,+\mathrm{z}-1 / 2$ & & &
\end{tabular}

Table 4. Supramolecular bond distances and angles $\left[\AA^{\AA},{ }^{\circ}\right]$ for $\mathbf{5} \cdot \mathbf{D M S O}$.

\begin{tabular}{ccc}
\hline & Experimental & Calculated \\
\hline $\mathrm{Te}(1)-\mathrm{N}(2)^{\mathrm{a}}$ & $2.744(4)$ & 2.728 \\
$\mathrm{~N}(2)-\mathrm{Te}(1)-\mathrm{N}(2)^{\mathrm{a}}$ & $70.2(2)$ & 70.7 \\
$\mathrm{~N}(1)-\mathrm{Te}(1)-\mathrm{N}(2)^{\mathrm{a}}$ & $155.3(2)$ & 155.0 \\
$\mathrm{Te}(1)-\mathrm{O}(1)$ & $2.834(5)$ & 2.737 \\
$\mathrm{~N}(1)-\mathrm{Te}(1)-\mathrm{O}(1)$ & $78.3(2)$ & 79.8 \\
$\mathrm{~N}(2)-\mathrm{Te}(1)-\mathrm{O}(1)$ & $163.0(1)$ & 164.1 \\
$\mathrm{C}(1)-\mathrm{N}(1)-\mathrm{Te}(1)-\mathrm{O}(1)$ & $175.6(4)$ & 178.2 \\
$\mathrm{C}(1)-\mathrm{N}(1)-\mathrm{Te}(1)-\mathrm{S}(1)$ & $169.1(4)$ & 170.5 \\
${ }^{\mathrm{a}}-\mathrm{x}+1,-\mathrm{y}+2,-\mathrm{z}$ & &
\end{tabular}


Table S5. DFT optimized coordinates for $4 \mathbf{c}$

\begin{tabular}{cccc}
\hline & $\mathbf{x}$ & $\mathbf{y}$ & $\mathbf{z}$ \\
\hline $\mathrm{H}$ & 0.0000 & -1.2455 & 3.4996 \\
$\mathrm{H}$ & 0.0000 & -2.5223 & 1.3701 \\
$\mathrm{H}$ & 0.0000 & 2.5223 & 1.3701 \\
$\mathrm{H}$ & 0.0000 & 1.2455 & 3.4996 \\
$\mathrm{C}$ & 0.0000 & -0.7171 & 2.5454 \\
$\mathrm{C}$ & 0.0000 & -1.4329 & 1.3771 \\
$\mathrm{C}$ & 0.0000 & -0.7416 & 0.1173 \\
$\mathrm{C}$ & 0.0000 & 0.7416 & 0.1173 \\
$\mathrm{C}$ & 0.0000 & 1.4329 & 1.3771 \\
$\mathrm{C}$ & 0.0000 & 0.7171 & 2.5454 \\
$\mathrm{~N}$ & 0.0000 & 1.4082 & -1.0364 \\
$\mathrm{~N}$ & 0.0000 & -1.4082 & -1.0364 \\
$\mathrm{Te}$ & 0.0000 & 0.0000 & -2.4869
\end{tabular}

Table S6. DFT optimized coordinates for $\mathbf{4} \mathbf{c}_{2}$

\begin{tabular}{cccc}
\hline & $\mathbf{x}$ & $\mathbf{y}$ & $\mathbf{z}$ \\
\hline $\mathrm{H}$ & 5.8312 & -0.0325 & 0.0000 \\
$\mathrm{H}$ & 3.5892 & -1.0630 & 0.0000 \\
$\mathrm{H}$ & 4.1385 & 3.9438 & 0.0000 \\
$\mathrm{H}$ & 6.1180 & 2.4353 & 0.0000 \\
$\mathrm{H}$ & -6.1180 & -2.4353 & 0.0000 \\
$\mathrm{H}$ & -4.1385 & -3.9438 & 0.0000 \\
$\mathrm{H}$ & -3.5892 & 1.0630 & 0.0000 \\
$\mathrm{H}$ & -5.8312 & 0.0325 & 0.0000 \\
$\mathrm{C}$ & 4.9439 & 0.5995 & 0.0000 \\
$\mathrm{C}$ & 3.7041 & 0.0190 & 0.0000 \\
$\mathrm{C}$ & 2.5317 & 0.8451 & 0.0000 \\
$\mathrm{C}$ & 2.6952 & 2.3217 & 0.0000 \\
$\mathrm{C}$ & 4.0288 & 2.8608 & 0.0000 \\
$\mathrm{C}$ & 5.1097 & 2.0230 & 0.0000 \\
$\mathrm{C}$ & -5.1097 & -2.0230 & 0.0000 \\
$\mathrm{C}$ & -4.0288 & -2.8608 & 0.0000 \\
$\mathrm{C}$ & -2.6952 & -2.3217 & 0.0000 \\
$\mathrm{C}$ & -2.5317 & -0.8451 & 0.0000 \\
$\mathrm{C}$ & -3.7041 & -0.0191 & 0.0000 \\
$\mathrm{C}$ & -4.9439 & -0.5995 & 0.0000 \\
$\mathrm{~N}$ & 1.6223 & 3.0920 & 0.0000 \\
$\mathrm{~N}$ & 1.3018 & 0.3564 & 0.0000 \\
$\mathrm{~N}$ & -1.3018 & -0.3564 & 0.0000 \\
$\mathrm{~N}$ & -1.6223 & -3.0921 & 0.0000 \\
$\mathrm{Te}$ & -0.0370 & 1.9066 & 0.0000 \\
$\mathrm{Te}$ & 0.0370 & -1.9066 & 0.0000
\end{tabular}

Table S7. DFT optimized coordinates for $\mathbf{4} \mathbf{c}_{\mathbf{6}}$

\begin{tabular}{cccc}
\hline & $\mathbf{x}$ & $\mathbf{y}$ & $\mathbf{z}$ \\
\hline $\mathrm{H}$ & 5.8190 & -0.6346 & 0.0000 \\
$\mathrm{H}$ & 3.4647 & -1.4002 & 0.0000 \\
$\mathrm{H}$ & 4.6000 & 3.5048 & 0.0000 \\
$\mathrm{H}$ & 6.3782 & 1.7832 & 0.0000 \\
$\mathrm{H}$ & -6.3782 & -1.7832 & 0.0000 \\
$\mathrm{H}$ & -4.6000 & -3.5048 & 0.0000 \\
$\mathrm{H}$ & -3.4647 & 1.4002 & 0.0000 \\
$\mathrm{H}$ & -5.8190 & 0.6345 & 0.0000 \\
$\mathrm{H}$ & 7.6131 & 6.7745 & 0.0000 \\
$\mathrm{H}$ & 5.2527 & 6.0437 & 0.0000 \\
$\mathrm{H}$ & 6.4492 & 10.9360 & 0.0000 \\
$\mathrm{H}$ & 8.2147 & 9.1838 & 0.0000 \\
$\mathrm{H}$ & -4.6040 & 5.8974 & 0.0000 \\
$\mathrm{H}$ & -2.8582 & 4.1398 & 0.0000 \\
$\mathrm{H}$ & -1.6259 & 9.0203 & 0.0000 \\
$\mathrm{H}$ & -3.9924 & 8.3040 & 0.0000 \\
$\mathrm{H}$ & 3.9924 & -8.3040 & 0.0000 \\
$\mathrm{H}$ & 1.6259 & -9.0203 & 0.0000 \\
$\mathrm{H}$ & 2.8582 & -4.1398 & 0.0000 \\
$\mathrm{H}$ & 4.6040 & -5.8974 & 0.0000 \\
$\mathrm{H}$ & -8.2147 & -9.1838 & 0.0000 \\
$\mathrm{H}$ & -6.4493 & -10.9360 & 0.0000 \\
$\mathrm{H}$ & -5.2527 & -6.0437 & 0.0000 \\
$\mathrm{H}$ & -7.6131 & -6.7745 & 0.0000 \\
$\mathrm{C}$ & 5.0068 & 0.0915 & 0.0000 \\
$\mathrm{C}$ & 3.7077 & -0.3387 & 0.0000 \\
$\mathrm{C}$ & 2.6338 & 0.6157 & 0.0000 \\
$\mathrm{C}$ & 2.9672 & 2.0589 & 0.0000 \\
$\mathrm{C}$ & -0.8330 & 6.9885 & 0.0000 \\
$\mathrm{C}$ & 4.3516 & 2.4445 & 0.0000 \\
$\mathrm{C}$ & 5.3298 & 1.4876 & 0.0000 \\
$\mathrm{C}$ & -5.3298 & -1.4876 & 0.0000 \\
$\mathrm{C}$ & -4.3516 & -2.4445 & 0.0000 \\
$\mathrm{C}$ & -3.5496 & 6.1716 & 0.0000 \\
$\mathrm{C}$ & -2.9672 & -2.0589 & 0.0000 \\
$\mathrm{C}$ & -2.6338 & -0.6157 & 0.0000 \\
$\mathrm{C}$ & -3.7077 & 0.3387 & 0.0000 \\
$\mathrm{C}$ & -5.0068 & -0.0915 & 0.0000 \\
$\mathrm{C}$ & 6.8144 & 7.5151 & 0.0000 \\
$\mathrm{C}$ & 5.5099 & 7.1017 & 0.0000 \\
$\mathrm{C}$ & 4.4540 & 8.0729 & 0.0000 \\
$\mathrm{C}$ & 4.8052 & 9.5144 & 0.0000 \\
$\mathrm{C}$ & 6.1976 & 9.8770 & 0.0000 \\
$\mathrm{C}$ & & & \\
$\mathrm{C}$ & -2.5912 & 5.1955 & 0.0000 \\
$\mathrm{H}$ & -.1989 & 5.5506 & 0.0000 \\
$\mathrm{H}$ & &
\end{tabular}


Table S7. DFT optimized coordinates for $\mathbf{4} \mathbf{c}_{\mathbf{6}}$ (continued)

\begin{tabular}{cccc}
\hline & $\mathbf{x}$ & $\mathbf{y}$ & $\mathbf{z}$ \\
\hline $\mathrm{C}$ & -1.8881 & 7.9640 & 0.0000 \\
$\mathrm{C}$ & -3.1961 & 7.5602 & 0.0000 \\
$\mathrm{C}$ & 3.1961 & -7.5602 & 0.0000 \\
$\mathrm{C}$ & 1.8881 & -7.9640 & 0.0000 \\
$\mathrm{C}$ & 0.8330 & -6.9885 & 0.0000 \\
$\mathrm{C}$ & 1.1989 & -5.5506 & 0.0000 \\
$\mathrm{C}$ & 2.5912 & -5.1955 & 0.0000 \\
$\mathrm{C}$ & 3.5496 & -6.1716 & 0.0000 \\
$\mathrm{C}$ & -7.1613 & -8.9057 & 0.0000 \\
$\mathrm{C}$ & -6.1976 & -9.8770 & 0.0000 \\
$\mathrm{C}$ & -4.8052 & -9.5144 & 0.0000 \\
$\mathrm{C}$ & -4.4540 & -8.0729 & 0.0000 \\
$\mathrm{C}$ & -5.5099 & -7.1017 & 0.0000 \\
$\mathrm{C}$ & -6.8144 & -7.5151 & 0.0000 \\
$\mathrm{~N}$ & 1.9699 & 2.9267 & 0.0000 \\
$\mathrm{~N}$ & 1.3570 & 0.2727 & 0.0000 \\
$\mathrm{~N}$ & -1.3570 & -0.2727 & 0.0000 \\
$\mathrm{~N}$ & -1.9699 & -2.9267 & 0.0000 \\
$\mathrm{~N}$ & 3.8366 & 10.4150 & 0.0000 \\
$\mathrm{~N}$ & 3.1738 & 7.7365 & 0.0000 \\
$\mathrm{~N}$ & 0.4531 & 7.2986 & 0.0000 \\
$\mathrm{~N}$ & -0.2276 & 4.6546 & 0.0000 \\
$\mathrm{~N}$ & 0.2276 & -4.6546 & 0.0000 \\
$\mathrm{~N}$ & -0.4531 & -7.2986 & 0.0000 \\
$\mathrm{~N}$ & -3.1738 & -7.7365 & 0.0000 \\
$\mathrm{~N}$ & -3.8366 & -10.4150 & 0.0000 \\
$\mathrm{Te}$ & 0.1646 & 1.9454 & 0.0000 \\
$\mathrm{Te}$ & -0.1646 & -1.9454 & 0.0000 \\
$\mathrm{Te}$ & 2.0450 & 9.4364 & 0.0000 \\
$\mathrm{Te}$ & 1.6019 & 5.5869 & 0.0000 \\
$\mathrm{Te}$ & -1.6019 & -5.5869 & 0.0000 \\
$\mathrm{Te}$ & -2.0450 & -9.4364 & 0.0000 \\
& & &
\end{tabular}

Table S8. DFT optimized coordinates for 5

\begin{tabular}{cccc}
\hline & $\mathbf{x}$ & $\mathbf{y}$ & $\mathbf{z}$ \\
\hline $\mathrm{H}$ & 0.2855 & 3.0483 & -3.2377 \\
$\mathrm{H}$ & 0.1462 & 1.4510 & -1.3465 \\
$\mathrm{C}$ & 0.3005 & 3.4336 & -2.2214 \\
$\mathrm{C}$ & 0.2227 & 2.5140 & -1.1328 \\
$\mathrm{C}$ & 0.3449 & 4.3478 & 0.4695 \\
$\mathrm{C}$ & 0.2439 & 2.9473 & 0.1645 \\
$\mathrm{C}$ & 0.4118 & 5.3117 & -0.6701 \\
$\mathrm{C}$ & 0.3918 & 4.7812 & -2.0059 \\
$\mathrm{~N}$ & 0.5005 & 6.6103 & -0.4220 \\
$\mathrm{~N}$ & 0.3747 & 4.8047 & 1.7130 \\
$\mathrm{Br}$ & 0.4852 & 5.9926 & -3.4824 \\
$\mathrm{Br}$ & 0.1468 & 1.6922 & 1.6039 \\
$\mathrm{Te}$ & 0.5132 & 6.8037 & 1.5765
\end{tabular}

Table S9. DFT optimized coordinates for $\mathbf{5}_{2}$

\begin{tabular}{cccc} 
& $\mathbf{x}$ & $\mathbf{y}$ & $\mathbf{z}$ \\
\hline $\mathrm{H}$ & 0.0200 & 5.8897 & 0.0000 \\
$\mathrm{H}$ & 2.4851 & 6.1521 & 0.0000 \\
$\mathrm{H}$ & -0.0200 & -5.8897 & 0.0000 \\
$\mathrm{H}$ & -2.4851 & -6.1521 & 0.0000 \\
$\mathrm{C}$ & 0.6436 & 4.9992 & 0.0000 \\
$\mathrm{C}$ & 0.8463 & 2.5660 & 0.0000 \\
$\mathrm{C}$ & 0.0595 & 3.7618 & 0.0000 \\
$\mathrm{C}$ & 2.8769 & 4.0547 & 0.0000 \\
$\mathrm{C}$ & 2.3343 & 2.7191 & 0.0000 \\
$\mathrm{C}$ & 2.0607 & 5.1514 & 0.0000 \\
$\mathrm{C}$ & -0.6436 & -4.9992 & 0.0000 \\
$\mathrm{C}$ & -0.8463 & -2.5660 & 0.0000 \\
$\mathrm{C}$ & -0.0595 & -3.7618 & 0.0000 \\
$\mathrm{C}$ & -2.8769 & -4.0547 & 0.0000 \\
$\mathrm{C}$ & -2.3344 & -2.7191 & 0.0000 \\
$\mathrm{C}$ & -2.0607 & -5.1514 & 0.0000 \\
$\mathrm{~N}$ & 0.3572 & 1.3354 & 0.0000 \\
$\mathrm{~N}$ & 3.0835 & 1.6388 & 0.0000 \\
$\mathrm{~N}$ & -0.3572 & -1.3354 & 0.0000 \\
$\mathrm{~N}$ & -3.0835 & -1.6388 & 0.0000 \\
$\mathrm{Br}$ & -1.8613 & 3.6495 & 0.0000 \\
$\mathrm{Br}$ & 4.7743 & 4.2685 & 0.0000 \\
$\mathrm{Br}$ & 1.8613 & -3.6495 & 0.0000 \\
$\mathrm{Br}$ & -4.7743 & -4.2685 & 0.0000 \\
$\mathrm{Te}$ & 1.8981 & -0.0022 & 0.0000 \\
$\mathrm{Te}$ & -1.8981 & 0.0022 & 0.0000
\end{tabular}


Table S10. DFT optimized coordinates for $\mathbf{5}_{\mathbf{3}}$

\begin{tabular}{|c|c|c|c|}
\hline & $\mathbf{x}$ & $\mathbf{y}$ & $\mathbf{z}$ \\
\hline $\mathrm{H}$ & 5.8691 & 1.1462 & 0.0000 \\
\hline $\mathrm{H}$ & 5.8349 & 3.6251 & 0.0000 \\
\hline $\mathrm{H}$ & -5.7858 & -2.8599 & 0.0000 \\
\hline $\mathrm{H}$ & -5.8256 & -0.3829 & 0.0000 \\
\hline $\mathrm{H}$ & 5.2951 & -9.1326 & 0.0000 \\
\hline $\mathrm{H}$ & 6.1145 & -6.7916 & 0.0000 \\
\hline $\mathrm{C}$ & 4.9100 & 1.6588 & 0.0000 \\
\hline $\mathrm{C}$ & 3.7514 & 0.9303 & 0.0000 \\
\hline $\mathrm{C}$ & 2.4703 & 1.5711 & 0.0000 \\
\hline $\mathrm{C}$ & 2.4426 & 3.0657 & 0.0000 \\
\hline $\mathrm{C}$ & 3.7055 & 3.7651 & 0.0000 \\
\hline $\mathrm{C}$ & 4.8912 & 3.0849 & 0.0000 \\
\hline $\mathrm{C}$ & -4.8426 & -2.3187 & 0.0000 \\
\hline $\mathrm{C}$ & -3.6561 & -2.9991 & 0.0000 \\
\hline $\mathrm{C}$ & -2.3898 & -2.3102 & 0.0000 \\
\hline $\mathrm{C}$ & -2.4212 & -0.8131 & 0.0000 \\
\hline $\mathrm{C}$ & -3.7049 & -0.1698 & 0.0000 \\
\hline $\mathrm{C}$ & -4.8655 & -0.8941 & 0.0000 \\
\hline $\mathrm{C}$ & 4.5711 & -8.3212 & 0.0000 \\
\hline $\mathrm{C}$ & 3.2310 & -8.5927 & 0.0000 \\
\hline $\mathrm{C}$ & 2.2561 & -7.5313 & 0.0000 \\
\hline $\mathrm{C}$ & 2.7490 & -6.1194 & 0.0000 \\
\hline $\mathrm{C}$ & 4.1726 & -5.9202 & 0.0000 \\
\hline $\mathrm{C}$ & 5.0426 & -6.9752 & 0.0000 \\
\hline $\mathrm{N}$ & 1.2794 & 3.6815 & 0.0000 \\
\hline $\mathrm{N}$ & 1.3102 & 0.9298 & 0.0000 \\
\hline $\mathrm{N}$ & -1.2639 & -0.1703 & 0.0000 \\
\hline $\mathrm{N}$ & -1.2157 & -2.9137 & 0.0000 \\
\hline $\mathrm{N}$ & 1.8752 & -5.1215 & 0.0000 \\
\hline $\mathrm{N}$ & 0.9538 & -7.7580 & 0.0000 \\
\hline $\mathrm{Br}$ & 3.8432 & -0.9856 & 0.0000 \\
\hline $\mathrm{Br}$ & 3.6940 & 5.6757 & 0.0000 \\
\hline $\mathrm{Br}$ & -3.6844 & -4.9142 & 0.0000 \\
\hline $\mathrm{Br}$ & -3.8212 & 1.7481 & 0.0000 \\
\hline $\mathrm{Br}$ & 2.6237 & -10.4078 & 0.0000 \\
\hline $\mathrm{Br}$ & 4.8858 & -4.1404 & 0.0000 \\
\hline $\mathrm{Te}$ & -0.2013 & 2.2993 & 0.0000 \\
\hline $\mathrm{Te}$ & 0.2672 & -1.5131 & 0.0000 \\
\hline $\mathrm{Te}$ & 0.0318 & -5.9629 & 0.0000 \\
\hline
\end{tabular}

Table S11. DFT optimized coordinates for 5-5 (Br-Br SBI)

\begin{tabular}{cccc}
\hline & $\mathbf{x}$ & $\mathbf{y}$ & $\mathbf{z}$ \\
\hline $\mathrm{H}$ & -2.5230 & 7.7637 & -4.8445 \\
$\mathrm{H}$ & -2.2220 & 5.3638 & -4.2886 \\
$\mathrm{H}$ & -0.4602 & -2.1360 & -0.3054 \\
$\mathrm{H}$ & -0.7397 & -4.5348 & -0.8743 \\
$\mathrm{C}$ & -2.0306 & 7.4964 & -3.9133 \\
$\mathrm{C}$ & -0.9385 & 8.1484 & -1.8315 \\
$\mathrm{C}$ & -1.5880 & 8.4775 & -3.0702 \\
$\mathrm{C}$ & -1.2424 & 5.7305 & -2.4374 \\
$\mathrm{C}$ & -0.7572 & 6.7038 & -1.4978 \\
$\mathrm{C}$ & -1.8576 & 6.1172 & -3.5948 \\
$\mathrm{C}$ & -0.9465 & -2.4040 & -1.2398 \\
$\mathrm{C}$ & -2.0333 & -1.7447 & -3.3229 \\
$\mathrm{C}$ & -1.3924 & -1.4209 & -2.0773 \\
$\mathrm{C}$ & -1.7179 & -4.1661 & -2.7283 \\
$\mathrm{C}$ & -2.2053 & -3.1892 & -3.6623 \\
$\mathrm{C}$ & -1.1088 & -3.7830 & -1.5667 \\
$\mathrm{~N}$ & -0.5155 & 9.0732 & -0.9834 \\
$\mathrm{~N}$ & -0.1771 & 6.3636 & -0.3575 \\
$\mathrm{~N}$ & -2.4570 & -0.8199 & -4.1705 \\
$\mathrm{~N}$ & -2.7816 & -3.5295 & -4.8048 \\
$\mathrm{Br}$ & -1.8282 & 10.3200 & -3.5245 \\
$\mathrm{Br}$ & -1.0080 & 3.8725 & -2.0671 \\
$\mathrm{Br}$ & -1.1773 & 0.4106 & -1.5816 \\
$\mathrm{Br}$ & -1.9305 & -6.0215 & -3.1416 \\
$\mathrm{Te}$ & 0.2770 & 8.0859 & 0.5811 \\
$\mathrm{Te}$ & -3.2390 & -1.8087 & -5.7412 \\
& & &
\end{tabular}


Table S12. DFT optimized coordinates for 5-5 (Te-Br SBI)

\begin{tabular}{cccc} 
& $\mathbf{x}$ & $\mathbf{y}$ & $\mathbf{z}$ \\
\hline $\mathrm{H}$ & -2.4972 & 7.7496 & -4.8528 \\
$\mathrm{H}$ & -2.2244 & 5.3488 & -4.2953 \\
$\mathrm{H}$ & 0.4666 & 2.1010 & 0.2819 \\
$\mathrm{H}$ & 0.7215 & 4.4959 & 0.8243 \\
$\mathrm{C}$ & -2.0146 & 7.4771 & -3.9178 \\
$\mathrm{C}$ & -0.9300 & 8.1137 & -1.8259 \\
$\mathrm{C}$ & -1.5727 & 8.4517 & -3.0675 \\
$\mathrm{C}$ & -1.2522 & 5.7036 & -2.4352 \\
$\mathrm{C}$ & -0.7549 & 6.6679 & -1.4941 \\
$\mathrm{C}$ & -1.8551 & 6.0962 & -3.5975 \\
$\mathrm{C}$ & 0.9520 & 2.3719 & 1.2159 \\
$\mathrm{C}$ & 2.0363 & 1.7343 & 3.3071 \\
$\mathrm{C}$ & 1.3934 & 1.3971 & 2.0655 \\
$\mathrm{C}$ & 1.7081 & 4.1402 & 2.6901 \\
$\mathrm{C}$ & 2.2047 & 3.1806 & 3.6354 \\
$\mathrm{C}$ & 1.1059 & 3.7575 & 1.5268 \\
$\mathrm{~N}$ & -0.5092 & 9.0307 & -0.9701 \\
$\mathrm{~N}$ & -0.1709 & 6.3289 & -0.3538 \\
$\mathrm{~N}$ & 2.4600 & 0.8174 & 4.1639 \\
$\mathrm{~N}$ & 2.7748 & 3.5323 & 4.7782 \\
$\mathrm{Br}$ & -1.8009 & 10.2961 & -3.5169 \\
$\mathrm{Br}$ & -1.0730 & 3.8322 & -2.0531 \\
$\mathrm{Br}$ & 1.1547 & -0.4490 & 1.6217 \\
$\mathrm{Br}$ & 1.9133 & 6.0053 & 3.1199 \\
$\mathrm{Te}$ & 0.2842 & 8.0519 & 0.5995 \\
$\mathrm{Te}$ & 3.2339 & 1.8178 & 5.7311
\end{tabular}

Table S13. DFT optimized coordinates for $\mathbf{5 - 5} \mathbf{5}_{2}-\mathbf{5}$

\begin{tabular}{cccc}
\hline & $\mathbf{x}$ & $\mathbf{y}$ & $\mathbf{z}$ \\
\hline $\mathrm{H}$ & 0.1638 & 5.8915 & 0.0000 \\
$\mathrm{H}$ & 2.6353 & 6.1038 & 0.0000 \\
$\mathrm{H}$ & -0.1638 & -5.8915 & 0.0000 \\
$\mathrm{H}$ & -2.6353 & -6.1038 & 0.0000 \\
$\mathrm{H}$ & 7.4619 & 2.1792 & 0.0000 \\
$\mathrm{H}$ & 5.4105 & 0.7989 & 0.0000 \\
$\mathrm{H}$ & -7.4619 & -2.1792 & 0.0000 \\
$\mathrm{H}$ & -5.4105 & -0.7989 & 0.0000 \\
$\mathrm{C}$ & 0.7731 & 4.9884 & 0.0000 \\
$\mathrm{C}$ & 0.9297 & 2.5499 & 0.0000 \\
$\mathrm{C}$ & 0.1659 & 3.7621 & 0.0000 \\
$\mathrm{C}$ & 2.9837 & 3.9966 & 0.0000 \\
$\mathrm{C}$ & 2.4189 & 2.6719 & 0.0000 \\
$\mathrm{C}$ & 2.1908 & 5.1093 & 0.0000 \\
$\mathrm{C}$ & -0.7731 & -4.9884 & 0.0000 \\
$\mathrm{C}$ & -0.9298 & -2.5499 & 0.0000 \\
$\mathrm{C}$ & -0.1659 & -3.7621 & 0.0000 \\
$\mathrm{C}$ & -2.9837 & -3.9966 & 0.0000 \\
$\mathrm{C}$ & -2.4189 & -2.6719 & 0.0000
\end{tabular}

Table S13. DFT optimized coordinates for $\mathbf{5 - 5} \mathbf{5}_{\mathbf{2}} \mathbf{- 5}$ (continued)

$\begin{array}{cccc}\mathrm{C} & -2.1908 & -5.1093 & 0.0000 \\ \mathrm{C} & 7.5654 & 1.0947 & 0.0000 \\ \mathrm{C} & 8.9697 & -0.9022 & 0.0000 \\ \mathrm{C} & 8.8095 & 0.5270 & 0.0000 \\ \mathrm{C} & 6.4687 & -1.0644 & 0.0000 \\ \mathrm{C} & 7.7338 & -1.7418 & 0.0000 \\ \mathrm{C} & 6.3813 & 0.2976 & 0.0000 \\ \mathrm{C} & -7.5654 & -1.0947 & 0.0000 \\ \mathrm{C} & -8.9697 & 0.9022 & 0.0000 \\ \mathrm{C} & -8.8095 & -0.5270 & 0.0000 \\ \mathrm{C} & -6.4687 & 1.0644 & 0.0000 \\ \mathrm{C} & -7.7339 & 1.7418 & 0.0000 \\ \mathrm{C} & -6.3813 & -0.2976 & 0.0000 \\ \mathrm{~N} & 0.4165 & 1.3291 & 0.0000 \\ \mathrm{~N} & 3.1427 & 1.5708 & 0.0000 \\ \mathrm{~N} & -0.4165 & -1.3291 & 0.0000 \\ \mathrm{~N} & -3.1427 & -1.5708 & 0.0000 \\ \mathrm{~N} & 10.1558 & -1.4895 & 0.0000 \\ \mathrm{~N} & 7.8379 & -3.0617 & 0.0000 \\ \mathrm{~N} & -10.1558 & 1.4895 & 0.0000 \\ \mathrm{~N} & -7.8379 & 3.0617 & 0.0000 \\ \mathrm{Br} & -1.7521 & 3.7071 & 0.0000 \\ \mathrm{Br} & 4.8862 & 4.1949 & 0.0000 \\ \mathrm{Br} & 1.7521 & -3.7071 & 0.0000 \\ \mathrm{Br} & -4.8862 & -4.1949 & 0.0000 \\ \mathrm{Br} & 10.3715 & 1.6303 & 0.0000 \\ \mathrm{Br} & 4.8811 & -2.1399 & 0.0000 \\ \mathrm{Br} & -10.3715 & -1.6303 & 0.0000 \\ \mathrm{Br} & -4.8811 & 2.1399 & 0.0000 \\ \mathrm{Te} & 1.9136 & -0.0395 & 0.0000 \\ \mathrm{Te} & -1.9136 & 0.0395 & 0.0000 \\ \mathrm{Te} & 9.8092 & -3.4682 & 0.0000 \\ \mathrm{Te} & -9.8092 & 3.4682 & 0.0000\end{array}$


Table S14. DFT optimized coordinates for $\underline{\mathbf{5}_{2} \cdot \mathrm{DMSO}_{2}}$

\begin{tabular}{|c|c|c|c|}
\hline & $\mathbf{x}$ & $\mathbf{y}$ & $\mathbf{z}$ \\
\hline $\mathrm{H}$ & -0.3478 & -4.8915 & -3.2743 \\
\hline $\mathrm{H}$ & -0.4369 & -6.4996 & -1.3858 \\
\hline $\mathrm{H}$ & -1.4963 & -3.9901 & 3.7063 \\
\hline $\mathrm{H}$ & -1.9914 & -3.2889 & 5.3049 \\
\hline $\mathrm{H}$ & -1.0696 & -4.8503 & 5.2263 \\
\hline $\mathrm{H}$ & 1.4515 & -2.2511 & 6.7464 \\
\hline $\mathrm{H}$ & 0.6728 & -3.8523 & 6.9764 \\
\hline $\mathrm{H}$ & -0.3265 & -2.3405 & 7.0131 \\
\hline $\mathrm{H}$ & 0.3478 & 4.8915 & 3.2743 \\
\hline $\mathrm{H}$ & 0.4369 & 6.4996 & 1.3858 \\
\hline $\mathrm{H}$ & 1.4963 & 3.9901 & -3.7063 \\
\hline $\mathrm{H}$ & 1.9914 & 3.2889 & -5.3049 \\
\hline $\mathrm{H}$ & 1.0696 & 4.8503 & -5.2264 \\
\hline $\mathrm{H}$ & -1.4515 & 2.2511 & -6.7464 \\
\hline $\mathrm{H}$ & -0.6728 & 3.8523 & -6.9765 \\
\hline $\mathrm{H}$ & 0.3265 & 2.3405 & -7.0131 \\
\hline $\mathrm{C}$ & -0.3151 & -4.5125 & -2.2559 \\
\hline $\mathrm{C}$ & -0.3681 & -5.4359 & -1.1719 \\
\hline $\mathrm{C}$ & -0.2418 & -3.5928 & 0.4390 \\
\hline $\mathrm{C}$ & -0.3358 & -4.9928 & 0.1212 \\
\hline $\mathrm{C}$ & -0.1825 & -2.6298 & -0.6995 \\
\hline $\mathrm{C}$ & -0.2265 & -3.1657 & -2.0306 \\
\hline $\mathrm{C}$ & -1.2368 & -3.8701 & 4.7632 \\
\hline $\mathrm{C}$ & 0.5446 & -2.8395 & 6.5746 \\
\hline $\mathrm{C}$ & 0.3151 & 4.5125 & 2.2559 \\
\hline $\mathrm{C}$ & 0.3681 & 5.4359 & 1.1719 \\
\hline $\mathrm{C}$ & 0.2418 & 3.5928 & -0.4390 \\
\hline $\mathrm{C}$ & 0.3358 & 4.9928 & -0.1212 \\
\hline $\mathrm{C}$ & 0.1825 & 2.6298 & 0.6994 \\
\hline $\mathrm{C}$ & 0.2264 & 3.1657 & 2.0306 \\
\hline $\mathrm{C}$ & 1.2368 & 3.8701 & -4.7632 \\
\hline $\mathrm{C}$ & -0.5446 & 2.8395 & -6.5746 \\
\hline $\mathrm{N}$ & -0.0961 & -1.3439 & -0.4072 \\
\hline $\mathrm{N}$ & -0.2059 & -3.1245 & 1.6710 \\
\hline $\mathrm{N}$ & 0.0961 & 1.3439 & 0.4072 \\
\hline $\mathrm{N}$ & 0.2059 & 3.1245 & -1.6710 \\
\hline $\mathrm{O}$ & -0.0003 & -1.5179 & 4.3231 \\
\hline $\mathrm{O}$ & 0.0003 & 1.5179 & -4.3231 \\
\hline $\mathrm{S}$ & 0.3325 & -2.9473 & 4.7642 \\
\hline$S$ & -0.3325 & 2.9473 & -4.7642 \\
\hline $\mathrm{Br}$ & -0.1628 & -1.9655 & -3.5280 \\
\hline $\mathrm{Br}$ & -0.4114 & -6.2670 & 1.5568 \\
\hline $\mathrm{Br}$ & 0.1628 & 1.9654 & 3.5280 \\
\hline $\mathrm{Br}$ & 0.4114 & 6.2670 & -1.5568 \\
\hline $\mathrm{Te}$ & -0.0569 & -1.0944 & 1.6201 \\
\hline $\mathrm{Te}$ & 0.0568 & 1.0944 & -1.6201 \\
\hline
\end{tabular}

Table S15. DFT optimized coordinates for DMSO

\begin{tabular}{cccc}
\hline & $\mathbf{x}$ & $\mathbf{y}$ & $\mathbf{z}$ \\
\hline $\mathrm{H}$ & 2.0332 & 11.9690 & -3.7051 \\
$\mathrm{H}$ & 2.5382 & 11.2691 & -5.2833 \\
$\mathrm{H}$ & 1.6382 & 12.8418 & -5.2237 \\
$\mathrm{H}$ & -0.8945 & 10.3805 & -6.8650 \\
$\mathrm{H}$ & -0.0150 & 11.9385 & -7.0160 \\
$\mathrm{H}$ & 0.8935 & 10.3705 & -7.0555 \\
$\mathrm{C}$ & 1.7725 & 11.8554 & -4.7625 \\
$\mathrm{C}$ & 0.0364 & 10.9100 & -6.6377 \\
$\mathrm{O}$ & 0.4690 & 9.5254 & -4.3610 \\
$\mathrm{~S}$ & 0.1868 & 10.9369 & -4.8107
\end{tabular}

Table S16. DFT optimized coordinates for 7-5

\begin{tabular}{cccc}
\hline & $\mathbf{x}$ & $\mathbf{y}$ & $\mathbf{z}$ \\
\hline $\mathrm{H}$ & -2.5033 & 7.7552 & -4.8407 \\
$\mathrm{H}$ & -2.2372 & 5.3522 & -4.2946 \\
$\mathrm{H}$ & 1.8526 & 5.2335 & 2.9748 \\
$\mathrm{H}$ & 0.4711 & 2.1387 & 0.2891 \\
$\mathrm{H}$ & 0.7380 & 4.5218 & 0.8464 \\
$\mathrm{C}$ & -2.0161 & 7.4778 & -3.9097 \\
$\mathrm{C}$ & -0.9232 & 8.1049 & -1.8222 \\
$\mathrm{C}$ & -1.5665 & 8.4506 & -3.0609 \\
$\mathrm{C}$ & -1.2542 & 5.6940 & -2.4383 \\
$\mathrm{C}$ & -0.7545 & 6.6571 & -1.4967 \\
$\mathrm{C}$ & -1.8607 & 6.0953 & -3.5960 \\
$\mathrm{C}$ & 0.9591 & 2.4059 & 1.2239 \\
$\mathrm{C}$ & 2.0420 & 1.7520 & 3.3106 \\
$\mathrm{C}$ & 1.3961 & 1.4244 & 2.0679 \\
$\mathrm{C}$ & 1.7256 & 4.1817 & 2.7125 \\
$\mathrm{C}$ & 2.2134 & 3.1999 & 3.6370 \\
$\mathrm{C}$ & 1.1233 & 3.7878 & 1.5514 \\
$\mathrm{~N}$ & -0.4969 & 9.0201 & -0.9657 \\
$\mathrm{~N}$ & -0.1762 & 6.3160 & -0.3549 \\
$\mathrm{~N}$ & 2.4618 & 0.8312 & 4.1645 \\
$\mathrm{~N}$ & 2.7874 & 3.5422 & 4.7824 \\
$\mathrm{Br}$ & -1.7892 & 10.2936 & -3.5011 \\
$\mathrm{Br}$ & -1.0725 & 3.8277 & -2.0602 \\
$\mathrm{Br}$ & 1.1410 & -0.4163 & 1.6133 \\
$\mathrm{Te}$ & 0.2792 & 8.0292 & 0.5937 \\
$\mathrm{Te}$ & 3.2361 & 1.8233 & 5.7265
\end{tabular}

